\title{
CARACTERIZAÇÃO DE ERROS EXPERIMENTAIS EM CIRCUITOS ELETRÔNICOS NO LABORATÓRIO REMOTO VISIR+
}

\author{
Isabelli Sasdelli Tavares - isabelli.sasdelli@ hotmail.com \\ Instituto Federal de Santa Catarina - IFSC \\ Avenida Abraão João Francisco, no 3899 \\ 88307-303 - Itajaí - Santa Catarina
}

Gustavo Ribeiro da Costa Alves - gca@ isep.ipp.pt

Instituto Politécnico do Porto - ISEP

Rua Doutor António Bernardino de Almeida, $n^{\circ} 431$

4249-015 - Porto - Portugal

Wilson Valente Junior - wilson.valente@ifsc.edu.br

Instituto Federal de Santa Catarina - IFSC

Avenida Abraão João Francisco, no 3899

88307-303 - Itajaí - Santa Catarina

Luis Carlos Martinhago Schlichting - schlicht@ifsc.edu.br

Instituto Federal de Santa Catarina - IFSC

Avenida Mauro Ramos, $n^{\circ} 950$

88020-300 - Florianópolis - Santa Catarina

Resumo: Programas de simulação e plataformas de experimentação são importantes aliados do estudante e pesquisador para unir conhecimentos teóricos e práticos. Frequentemente, durante a realização dos experimentos com circuitos elétricos e eletrônicos, os alunos cometem erros, sejam estes por falta de atenção ou por não assimilar corretamente algum conteúdo. Como forma de aprimorar esses conhecimentos e ampliar a experimentação, laboratórios remotos como Virtual Instruments Systems in Reality (VISIR) ganham espaço. Com o objetivo de construir um tutorial online para o auxílio dos estudantes, este artigo apresenta possíveis erros ao analisar experimentos com o diodo, expandindo assim trabalhos anteriores já publicados acerca desta temática, relacionada com a utilização do VISIR.

Palavras-chave: VISIR. Erros experimentais. Eletrônica. Diodo. Laboratório remoto.

\section{INTRODUÇÃO}

Desde o ensino médio os conhecimentos básicos de circuitos elétricos são ensinados através da física. Nos cursos de graduação da área de engenharia elétrica esse ensinamento é consideravelmente expandido. Tanto para o ensino médio quanto para o ensino técnico profissionalizante, bem como para o ensino de graduação em engenharia, é de suma importância unir o conhecimento teórico com o prático e respectivas simulações e experimentações.

Para isso, ferramentas de simulação e de experimentação cumprem com o objetivo de representar circuitos elétricos e eletrônicos. Essas ferramentas podem ser da forma tradicional, 
quando há uma gama de possibilidades de instrumentação virtual de circuitos, com resultados apresentados da forma ideal ou não (dependendo da complexidade da simulação), como da forma não tradicional, conhecida também como forma de instrumentação remota de circuitos. Uma das plataformas que trabalha de forma remota com experimentos reais acontecendo à distância é o laboratório Virtual Instruments Systems In Reality (VISIR). Esses laboratórios de instrumentação remota apresentam-se como uma alternativa no que diz respeito à aplicação dos conceitos teóricos aprendidos, representando assim, uma autonomia por parte dos estudantes.

Como o VISIR se trata de um projeto em constante desenvolvimento, diversos trabalhos acerca do laboratório vêm sendo realizados. Inicialmente, foi abordada uma estrutura para interpretar erros experimentais realizados no VISIR, trazendo a teoria dos circuitos elétricos e aplicando-a em circuitos elétricos simples, compostos por um elemento passivo apenas, o resistor (ALVES et al, 2019).

Após, outros trabalhos começaram a ser desenvolvidos, com a aplicação de corrente alternada e inserção de um elemento reativo, o capacitor. $O$ foco foi em circuitos simples (1:1), no qual ora trabalhou-se somente com o resistor, ora somente com o capacitor (MAÇANEIRO, 2019).

Os trabalhos citados exploram possíveis erros que podem ser cometidos durante os experimentos no laboratório remoto, criando assim, a base para um tutorial online que pode ser aliado aos materiais de estudo do aluno ou pesquisador.

Como forma de explorar os recursos e componentes disponíveis no VISIR, iniciou-se o trabalho com o componente eletrônico mais básico possível, o diodo. Nestes experimentos também se investigou, pela primeira vez, o uso de dois multímetros digitais simultaneamente.

\section{CENÁRIO EDUCACIONAL}

\subsection{Conceitos Fundamentais de Eletrônica}

Os cursos de engenharia elétrica incluem, ao menos, uma disciplina na área de eletrônica (Projeto Pedagógico do Curso de Bacharel em Engenharia Elétrica; Currículo do Curso de Engenharia Elétrica; Licenciatura em Engenharia Eléctrica e Electrónica). Nessas disciplinas começa-se por conhecer o componente mais básico da eletrônica, o diodo, seguido do transistor e do amplificador operacional, sendo que a complexidade aumenta gradativamente ao longo do curso.

O diodo é um componente semicondutor básico de dois terminais que permite que a corrente flua em apenas um sentido e, dependendo do material e dopagem utilizada, os componentes apresentam ligeiras diferenças entre si (OLIVEIRA; SEDRA, 2005).

Pode ser classificado em diodos de sinal e diodos de potência, e é possível construir diversos circuitos como retificação de meia onda e onda completa, circuitos limitadores, etc. Enquanto os diodos de sinal permitem baixa corrente e alta frequência, os de potência atuam em corrente elétrica maior e podem ser aplicados em circuitos retificadores de alimentação não controlados ou semi-controlados (BRAGA, 2012).

Experimentos que utilizam combinações de diodos são também muito utilizados e um exemplo desta configuração múltipla é a ponte retificadora de diodos (HUSAIN, 2018). Há ainda o diodo zener, capaz de regular a tensão de fontes de alimentação, além de estar presente em muitas aplicações em que se necessita de uma tensão fixa (BRAGA, 2012).

Este trabalho se restringe especificamente ao diodo e suas experimentações, mas, como sequência de estudo dos componentes eletrônicos, há o transistor, um dispositivo semicondutor de três terminais utilizado para amplificar ou trocar sinais eletrônicos e potência elétrica. Por 
fim, expandindo a complexidade dos elementos semicondutores, há o amplificador operacional, um dispositivo que contém, no mínimo, 5 terminais (embora seja disponibilizado em circuitos integrados com 8 pinos) e é capaz de amplificar o sinal de entrada com elevados ganhos.

\subsection{Experimentos em Laboratórios Remotos}

Os laboratórios de instrumentação remota, também conhecidos como laboratórios online ou laboratórios não tradicionais, são uma ferramenta alternativa para aulas práticas, como forma de aplicar os conceitos teóricos aprendidos anteriormente, através de experimentações. Consiste em conduzir remotamente experimentos reais, enquanto o aluno ou pesquisador está geograficamente longe do laboratório real.

Como comparação às aplicações de simulação, os simuladores podem obter respostas muito próximas às obtidas analiticamente, entretanto em um experimento real, as respostas podem variar por diversos fatores externos, como variações de temperatura, influência de interferências eletromagnéticas (emissões radiadas e/ou conduzidas), além de outros fatores internos, que são característicos de cada circuito e componente eletrônico (não idealidades) (BRANCO et al, 2017).

\subsection{Virtual Instruments Systems in Reality (VISIR)}

O VISIR+ é um projeto que vendo sendo desenvolvido em parceria com 12 instituições de ensino, localizadas em 6 países diferentes, em dois continentes, Europa e América do Sul, cofundado pelo programa Erasmus, da União Europeia. Tem como objetivo difundir e desenvolver o uso de laboratórios remotos, contribuindo assim para os cursos STEAM (Science, Technology, Engineering, Arts and Mathematics) nessas plataformas, o que permite maior autonomia dos estudantes em seus estudos, por meio de uma abordagem baseada em investigação (ABOUT VISIR, online).

O laboratório remoto VISIR, para experiências remotas com circuitos elétricos e eletrônicos, pretende ser uma réplica de um laboratório real, do Instituto Tecnológico de Blekinge (Blekinge Tekniska Hogskola, BTH), Suécia, adaptado para os mesmos tipos de experiências e de procedimentos laboratoriais presenciais (TEACHER'S GUIDE TO THE VISIR, online).

Como se trata de um laboratório remoto, alguns experimentos estão previamente preparados, outros precisam ser informados a uma equipe responsável, assim as instruções para o instrutor virtual são criadas em conjunto pelos professores e pelos técnicos de laboratório. Essas instruções são para a criação das max lists, ou seja, para fazer o contato dos componentes no laboratório físico, com base na component list existente (TEACHER'S GUIDE TO THE VISIR, online; COMPONENT LIST, online).

Sendo assim, o VISIR tem sido amplamente utilizado em várias pesquisas acadêmicas e aulas e práticas diárias de ensino (KULESZA et al, 2017).

\section{ERROS EXPERIMENTAIS EM UM LABORATÓRIO REMOTO}

Uma parte importante para reconhecer erros experimentais em um laboratório remoto é saber identificar o que são erros e quais tipos de erros são levados em consideração. O objetivo da medição na abordagem de erro é determinar uma estimativa do valor verdadeiro que esteja tão próxima quanto possível deste valor verdadeiro único. O desvio do valor verdadeiro é composto de dois tipos de erros: os aleatórios e os sistemáticos. Para efeito de laboratórios de 
ensino, consideram-se ainda os erros de procedimento, inerentes ao processo de experimentação e aprendizagem do aluno, que podem assumir duas naturezas: erros manuais e erros conceituais (VOCABULÁRIO INTERNACIONAL DE METROLOGIA, 2012).

Os erros manuais são erros acidentais, oriundos de falta de atenção, e não por assimilação do conteúdo. Tais erros podem ser observados em casos em que o aluno acidentalmente deixa um circuito aberto ou causa um curto-circuito, ou ainda por não configurar corretamente algum instrumento da bancada remota, como fonte de tensão e gerador de funções, ou os instrumentos de medição como multímetro e osciloscópio.

Já os erros conceituais são causados por definições mal construídas e/ou erros de interpretação, o que representa uma má assimilação do conteúdo e descaracteriza os fenômenos em estudo (JUNIOR, online). Esses erros podem ser, por exemplo, de má concepção do que é um circuito de associação em série ou paralelo, podendo inclusive acarretar danos ao circuito e/ou aos instrumentos envolvidos.

Um trabalho anterior abordou erros em circuitos com resistor, indutor e capacitor, explorando configurações série e paralelo, aplicação de corrente alternada e uso do osciloscópio (MENDONÇA et al, 2020). Já esta pesquisa reúne erros já levantados anteriormente, expandindo-os para o componente eletrônico diodo e explorando o uso de dois multímetros simultaneamente. Sendo assim, a concepção desses erros é parte fundamental na construção de um fiel tutorial online.

\section{METODOLOGIA}

A pesquisa foi desenvolvida no Instituto Superior de Engenharia de Porto (ISEP), através do programa de cooperação internacional PROPICIE e os experimentos foram realizados na plataforma VISIR instalada no Instituto Federal de Santa Catarina (IFSC).

$\mathrm{Na}$ Tabela 1 há as especificações padrões para as medições, considerando o experimento clássico do diodo, que consiste em levantar a curva característica de corrente versus tensão, abordando o uso de dois multímetros Minipa ET-2042. Para isso, foram necessários um resistor e uma fonte de tensão DC Agilent E3631A.

Tabela 1 - Parâmetros de medição estabelecidos.

\begin{tabular}{c|c|c}
\hline Resistor $(\Omega)$ & Tensão DC $(\mathrm{V})$ & Diodo \\
\hline $10 \mathrm{k}$ & 6 & $1 \mathrm{~N} 4007$ \\
\hline \multicolumn{2}{c}{ Fonte: Autores. }
\end{tabular}

\subsection{Elaboração dos erros}

Para fazer o levantamento dos erros, é necessário elaborar roteiros de interesse para a área, montar estes circuitos no laboratório remoto, listar os erros encontrados, tabelá-los com as devidas identificações e tirar fotografias a fim de registrar e documentar o experimento.

Como forma de explorar o uso dos multímetros digitais, montaram-se três tabelas, duas com a caracterização dos erros nos elementos do circuito (resistor e diodo) a partir do ponto de vista de um multímetro, e a outra com os erros oriundos do uso de dois multímetros.

Para entender a classificação dos erros e saber interpretá-los, todas as tabelas devem ser lidas da esquerda para a direita, de cima para baixo. No cruzamento das linhas e colunas, há os erros oriundos dessas leituras, identificados por "EXX", que se refere ao código do erro catalogado no VISIR, ou então pelo preenchimento de cor sólida da célula na tabela, que indica que todas as configurações estão corretas e a medição pode ser devidamente realizada. 
(C) COBENGE 2020

"Os desafios para formar hoje o engenheiro do amanhã"
$\mathrm{Ol} \mathrm{a} \mathrm{O3}$ de dezembro Evento On-line

Para as Tabelas 2, 3 e 4, a primeira coluna especifica o componente a ser analisado (resistor ou diodo), a segunda coluna indica se o gerador de funções ou fonte CC está ligada ou desligada (energia on e off).

Tabela 2 - Possíveis erros de leitura em um resistor utilizando um multímetro.

\begin{tabular}{|c|c|c|c|c|c|c|c|c|c|c|c|c|c|}
\hline \multicolumn{14}{|c|}{ Ao observar através de um multimetro } \\
\hline Componente & Energia & $\begin{array}{c}\text { Forma de } \\
\text { onda }\end{array}$ & $\begin{array}{l}\text { Conexão do } \\
\text { multimetro }\end{array}$ & Cabo & Vef Senoidal & Vef Quadrada & Vef Triangular & Aef Senoidal & Aef Quadrada & Aef Triangular & $\begin{array}{c}\text { Resistência } \\
\text { (n) }\end{array}$ & $\begin{array}{c}\text { Capacitância } \\
\text { (F) }\end{array}$ & Indutância $(\mathrm{H})$ \\
\hline \multirow{25}{*}{$\mathrm{R}$} & \multirow{13}{*}{ ON } & \multirow{4}{*}{ Senoide } & \multirow{2}{*}{ Serial } & $\mathrm{V} / \Omega$ & E1 & \multirow{4}{*}{ E18 } & \multirow{4}{*}{ E22 } & E3 & \multirow{4}{*}{ E20 } & \multirow{4}{*}{ E24 } & \multirow{13}{*}{ E9 } & \multirow{25}{*}{ E27 } & \multirow{25}{*}{ E26 } \\
\hline & & & & A & E2 & & & & & & & & \\
\hline & & & Paralelo & $\mathrm{V} / \Omega$ & & & & E4 & & & & & \\
\hline & & & & A & E5 & & & E6 & & & & & \\
\hline & & \multirow{4}{*}{ Quadrada } & \multirow{2}{*}{ Serial } & $\mathrm{V} / \Omega$ & \multirow{4}{*}{ E14 } & E1 & \multirow{4}{*}{ E23 } & \multirow{4}{*}{ E16 } & E3 & \multirow{4}{*}{ E25 } & & & \\
\hline & & & & A & & E2 & & & & & & & \\
\hline & & & \multirow{2}{*}{ Paralelo } & $\mathrm{V} / \Omega$ & & & & & E4 & & & & \\
\hline & & & & A & & E5 & & & E6 & & & & \\
\hline & & \multirow{4}{*}{ Triangular } & Serial & $\mathrm{V} / \Omega$ & \multirow{4}{*}{ E15 } & \multirow{4}{*}{ E19 } & E1 & \multirow{4}{*}{ E17 } & \multirow{4}{*}{ E21 } & E3 & & & \\
\hline & & & & A & & & E2 & & & & & & \\
\hline & & & \multirow{2}{*}{ Paralelo } & $\mathrm{V} / \Omega$ & & & & & & E4 & & & \\
\hline & & & & A & & & E5 & & & E6 & & & \\
\hline & & \multirow{5}{*}{ Senoide } & ência desaj & & \multicolumn{3}{|c|}{ E7 } & & & & & & \\
\hline & \multirow{12}{*}{ OFF } & & \multirow{2}{*}{ Serial } & $\mathrm{V} / \Omega$ & & & & & & & E10 & & \\
\hline & & & & A & & & & & & & E11 & & \\
\hline & & & Paralelo & $\mathrm{V} / \Omega$ & & & & & & & & & \\
\hline & & & 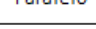 & A & & & & & & & E12 & & \\
\hline & & & Serial & $\mathrm{V} / \Omega$ & & & & & & & E10 & & \\
\hline & & Quadrada & entar & A & & E8 & & & $E_{13}$ & & E11 & & \\
\hline & & Qund & Paralelo & $\mathrm{V} / \Omega$ & & & & & 年 & & & & \\
\hline & & & (2) & A & & & & & & & E12 & & \\
\hline & & & Serial & $\mathrm{V} / \Omega$ & & & & & & & E10 & & \\
\hline & & Triangular & & A & & & & & & & E11 & & \\
\hline & & & Paralelo & $\mathrm{V} / \Omega$ & & & & & & & & & \\
\hline & & & 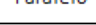 & A & & & & & & & E12 & & \\
\hline
\end{tabular}

Fonte: Autores.

Tabela 3 - Possíveis erros de leitura em um diodo utilizando um multímetro.

\begin{tabular}{|c|c|c|c|c|c|c|c|c|}
\hline \multicolumn{9}{|c|}{ Ao observar através de um multímetro } \\
\hline Componente & Energia & $\begin{array}{l}\text { Conexão do } \\
\text { multímetro }\end{array}$ & Cabo & Tensão & Corrente & 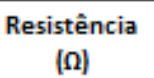 & $\begin{array}{l}\text { Capacitância } \\
\text { (F) }\end{array}$ & Indutância $(\mathrm{H})$ \\
\hline \multirow{8}{*}{ D } & \multirow{4}{*}{ ON } & \multirow{2}{*}{ Serial } & $\mathrm{V} / \Omega$ & E29 & E31 & \multirow{4}{*}{ E37 } & \multirow{8}{*}{ E43 } & \multirow{8}{*}{ E42 } \\
\hline & & & $\mathrm{A}$ & E30 & & & & \\
\hline & & \multirow{2}{*}{ Paralelo } & $\mathrm{V} / \Omega$ & & E32 & & & \\
\hline & & & $\mathrm{A}$ & E33 & E34 & & & \\
\hline & \multirow{4}{*}{ OFF } & \multirow{2}{*}{ Serial } & $\mathrm{V} / \Omega$ & \multirow{4}{*}{ E36 } & \multirow{4}{*}{ E41 } & E38 & & \\
\hline & & & A & & & E39 & & \\
\hline & & \multirow{2}{*}{ Paralelo } & $\mathrm{V} / \Omega$ & & & & & \\
\hline & & & $\mathrm{A}$ & & & E40 & & \\
\hline
\end{tabular}

Fonte: Autores.

Especificamente na Tabela 2, no caso do resistor, ao observar através de um multímetro, a terceira coluna diz respeito à forma de onda aplicada ao circuito e a quarta sobre a conexão das pontas de prova do multímetro. Isso aborda a perspectiva de outros experimentos possíveis de serem feitos com o diodo, como o caso de um retificador de meia onda com e sem filtro capacitivo, por isso a inserção das colunas referentes à corrente alternada. Há ainda as colunas relacionadas à leitura do medidor RLC HoldPeakHP-4070-L, em que o aluno pode cometer erros ao tentar ler a grandeza elétrica de maneira equivocada, por exemplo, ler resistência utilizando a função de medição da capacitância ou indutância. 
(C) COBENGE

"Os desafios para formar hoje o engenheiro do amanhã"
$\mathrm{Ol} \mathrm{a} \mathrm{O3}$ de dezembro Evento On-line

Tabela 4 - Possíveis erros de leitura utilizando dois

multímetros.

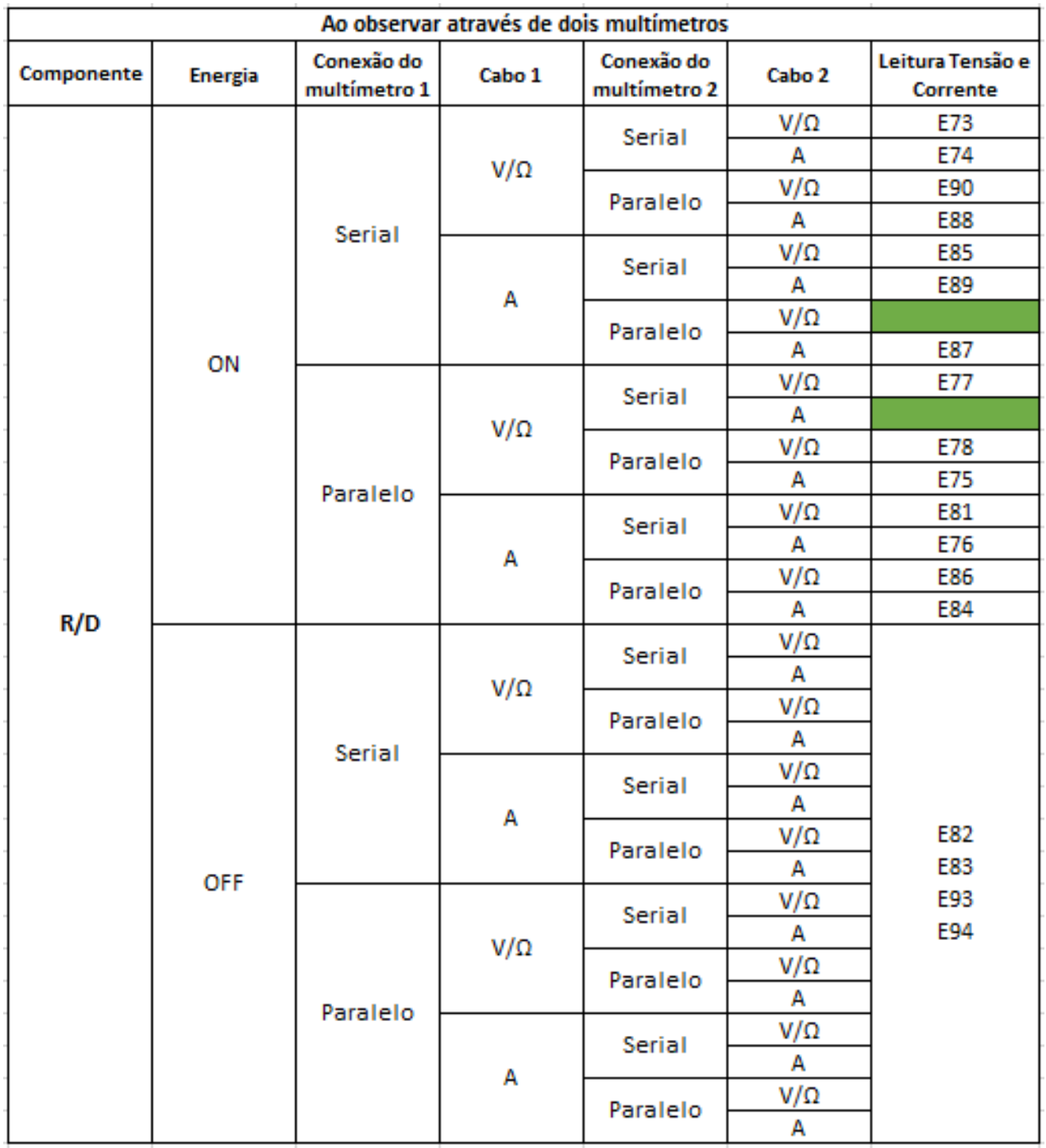

Fonte: Autores.

Para a Tabela 4, a de maior foco desta pesquisa, foi feita a análise a partir do ponto de vista de dois multímetros. No primeiro multímetro, operando como voltímetro, objetiva-se realizar a leitura da tensão no diodo, enquanto no segundo multímetro, operando como amperímetro, objetiva-se realizar a leitura de corrente, de modo a possibilitar o levantamento da curva característica do diodo (corrente versus tensão), facilitando assim a análise e ganhando tempo ao usar os dois multímetros simultaneamente. Esse experimento consiste, basicamente, em uma fonte de tensão $\mathrm{CC}$, um diodo e um resistor, além dos dois multímetros digitais.

No experimento de determinar a curva característica do diodo, foram considerados dois casos: desejando-se realizar leitura de tensão no multímetro 1 e de corrente no multímetro 2 e; desejando-se realizar leitura de corrente no multímetro 1 e de tensão no multímetro 2. Para isso, foram levantados erros relacionados à configuração incorreta do multímetro para a leitura desejada, por exemplo tentar medir tensão no diodo com o multímetro em série, como também erros relacionados ao uso incorreto das ponteiras (entradas $\mathrm{A}$ e $\mathrm{V} / \Omega$ ). 
Todos esses erros foram reproduzidos no VISIR e salvos como imagens, disponíveis na plataforma ResearchGate $($ uma rede social que visa o compartilhamento de informações entre pesquisadores e profissionais do meio científico, onde encontram-se também as tabelas e arquivos dos erros elencados e comentados (TAVARES, 2020).

Para melhor visualização da plataforma e de como um erro é tabelado, traz-se o erro E90, que diz respeito à tentativa de medir corrente no multímetro 1 e tensão no multímetro 2 , entretanto as pontas de prova do multímetro 1 estão conectadas com a entrada $\mathrm{V} / \Omega$, enquanto as configurações do multímetro 2 estão corretas. Isto indica um erro de leitura, mesmo que um dos multímetros esteja configurado corretamente, pois implica em uma leitura final indesejada, quando o objetivo é obter duas leituras corretas. A representação do erro E90 no laboratório VISIR e do circuito em um programa de simulação pode ser visto nas Figuras 1 e 2.

Figura 1 - Representação do erro 90 (E90) no VISIR.

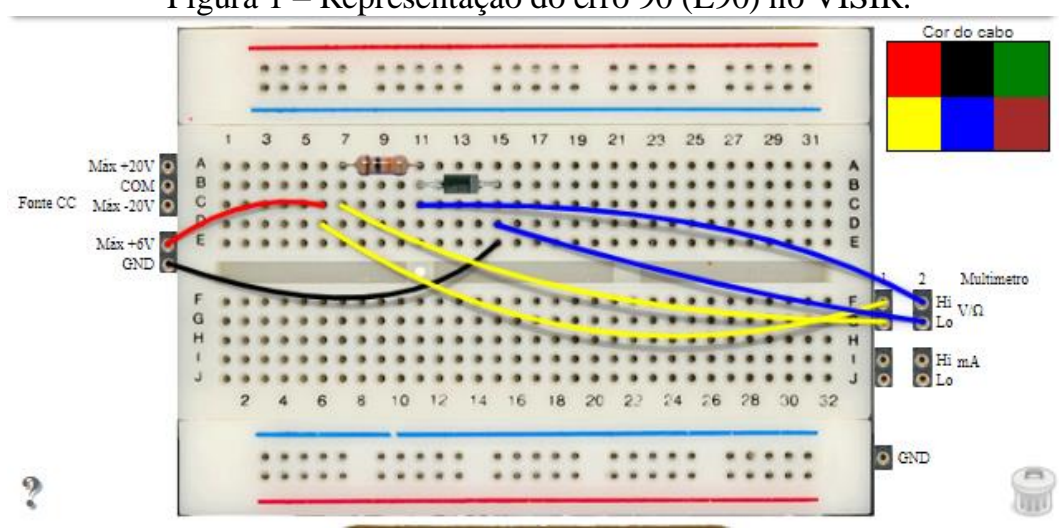

Fonte: Autores.

Figura 2 - Circuito da curva característica do diodo.

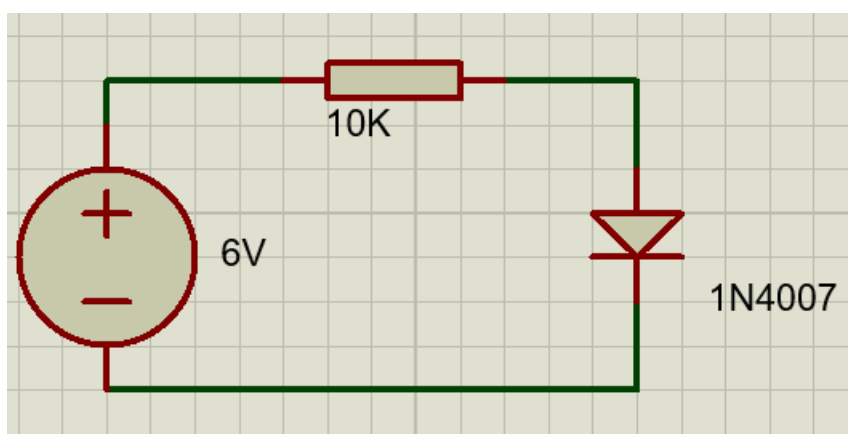

Fonte: Autores.

É possível observar que o multímetro 1, com conexões em amarelo, está conectado na configuração série e com as pontas de prova na entrada $\mathrm{V} / \Omega$ caracterizando um erro de leitura, e enquanto o multímetro 2, com conexões em azul, está conectado corretamente, em paralelo com o diodo para poder ler tensão e com as pontas de prova na entrada $\mathrm{V} / \Omega$.

\section{RESULTADOS}

A ampliação da caracterização dos erros para o uso de dois multímetros representa um aumento significativo na quantidade dos erros elencados e, principalmente, um avanço no que 
diz respeito à autonomia dos estudantes, à agilidade e à praticidade para realizar experimentos remotos com um tutorial online.

A Tabela 4 cresce de tal maneira que implica em uma abordagem computacional para ramificar ainda mais os erros manuais e conceituais abordados. Um erro manual não abordado na Tabela 4, fruto de desatenção por parte do aluno, é conectar as pontas de provas de ambos os multímetros na conexão de apenas um multímetro, ou seja, tentar ler tensão e corrente ao mesmo tempo a partir de um mesmo multímetro digital.

Expandir a pesquisa para experimentos que utilizam o diodo é de suma importância devido à variedade de aplicações com este componente eletrônico, abrindo espaço para demais componentes eletrônicos, como o transistor e o amplificador operacional, tal como citado anteriormente.

\section{CONCLUSÃO E TRABALHOS FUTUROS}

Com este estudo desenvolvido, pretende-se ampliar os trabalhos acerca de componentes eletrônicos como o diodo, abrindo espaço para projetos com utilização de dispositivos de três pinos, como os transistores, representando um avanço nos experimentos de circuitos elétricos e eletrônicos. Também abre espaço para estudo de circuitos mais complexos, com o uso simultâneo da fonte de tensão e gerador de funções para alimentar o circuito.

\section{Agradecimentos}

Agradeço primeiramente ao Instituto Federal de Santa Catarina (IFSC) pela oportunidade recebida através do programa de intercâmbio estudantil PROPICIE, e ao Instituto Superior de Engenharia de Porto (ISEP) pela pesquisa ofertada. Também agradeço ao Luis Schlichting e ao Daniel Dezan de Bona, que tornaram possível a realização desta pesquisa, auxiliando através do VISIR instalado no IFSC.

\section{REFERÊNCIAS}

About VISIR. Disponível em: https://visir.ifsc.edu.br/about/. Acesso em: 27 abr. 2020.

ALVES, G. R.; GARCÍA-ZUBÍA, J.; HERMÁNDEZ-JAVO, J.; CUADROS, V.;

SERRANO; FIDALGO, A. A framework for interpreting experimental errors in VISIR. In: 5th Experiment International Conference (exp.at'19), Portugal, v.1, n.1, p. 31-35, 2019.

BRAGA, N. C. Eletrônica Analógica. Volume 2, São Paulo: Instituto Newton Braga, 2012.

BRANCO, M. V; et al. Aspectos de Diferenciação entre Laboratórios Remotos e Simuladores. In: XLV Congresso Brasileiro de Educação em Engenharia, 2014, Joinville, Brasil. Anais. Joinville, 2017.

Component List: Relação de componentes e Circuitos disponíveis no Laboratório Remoto VISIR do IFSC. Disponível em: https://visir.florianopolis.ifsc.edu.br/visir/images/Visir_IFSC_21052018.pdf. Acesso em: 11 maio 2020.

Currículo do Curso de Engenharia Elétrica. Disponível em: http://cagr.sistemas.ufsc.br/relatorios/curriculoCurso?curso=202. Acesso em: 15 maio 2020. 
HUSAIN, M. A. DC Motor Control - A case study: Four Quadrant DC Motor Speed Coltro With Microcontroller. 2018. Disponível em:

https://books.google.pt/books?id=FPV6DwAAQBAJ. Acesso em: 11 maio 2020.

JUNIOR, J. S. S. Erros Conceituais. Disponível em:

https://mundoeducacao.uol.com.br/fisica/cinco-erros-conceituais-fisica.htm. Acesso em: 27 abr. 2020.

KULESZA, W. Kulesza et al. A federation of VISIR remote laboratories through the PILAR Project. In: 4th Experiment@international Conference (exp.at'17), 2017. IEEE, p.28-32.

Licenciatura em Engenharia Eléctrica e Electrónica. Disponível em:

https://www.ualg.pt/sites/ualg.pt/files/ise/Civil/Programas/programas_leee_pt.pdf. Acesso em: 15 maio 2020.

MAÇANEIRO, M. T.; Categorização de erros experimentais no VISIR com circuitos simples em corrente alternada. In: Seminário de Ensino, Pesquisa, Extensão e Inovação, 2019, Chapecó. Anais. Chapecó, 2019, pp. 870-872.

MENDONÇA, L. N.; MAÇANEIRO, M. T.; ALVES, G. R.; PIRES, D. S.; GARCÍAZUBÍA, J.; CUADROS, U.; HERNÁNDEZ-JAVO, V.; SERRANO. Classification of Experimental Errors Done in VISIR with Simple Alternated Current Circuits. In: 11th Global Engineering Education Conference (EDUCON), 2020, Porto, Portugal.

OLIVEIRA, C.; ZANETTI, H. Conceitos Fundamentais de Eletrônica. Disponível em: https://www.trilhas.iar.unicamp.br/curso/arduino/conceitoseletronica.pdf. Acesso em 15 maio 2020.

Projeto Pedagógico do Curso de Bacharel em Engenharia Elétrica. Disponível em: https://sig.ifsc.edu.br/sigaa/public/curso/ppp.jsf?lc=pt \_BR\&id=2398777. Acesso em: 14 maio 2020.

SEDRA, S.; SMITH, K. Microeletrônica. São Paulo: Editora Pearson Makron Books. 2005.

TAVARES, I. S. Diretório de arquivos VISIR. Disponível em: https://www.researchgate.net/project/Internship-at-ISEP-VISIR-2. Acesso em: 01 jun. 2020.

Teacher's Guide to the VISIR Online Laboratory for Electrical Experiments. Disponível em: http://openlabs.bth.se/static/Teacher \_manual\_6.pdf. Acesso em: 11 maio 2020.

VOCABULÁRIO INTERNACIONAL DE METROLOGIA (VIM): conceitos fundamentais e gerais e termos associados. Rio de Janeiro, 2012. 


\title{
CLASSIFICATION OF EXPERIMENTAL ERRORS IN ELECTRONIC CIRCUITS IN THE VISIR+ REMOTE LABORATORY
}

\begin{abstract}
Simulation programs and experimentation platforms are important allies for the student and researcher to unite theoretical and practical knowledge. Often, when conducting experiments with electrical and electronic circuits, students make mistakes, whether due to lack of attention or not correctly assimilating some content. As a way of improving this knowledge and expanding experimentation, remote laboratories such as Virtual Instruments Systems in Reality (VISIR) are gaining ground. In order to build an online tutorial to help students, this article presents possible mistakes when analyzing experiments with the diode, thus expanding previous work already published on this topic, related to the use of VISIR.
\end{abstract}

Keywords: VISIR. Experimental errors. Electronics. Diode. Remote laboratory. 